\title{
Comparative Three-Dimensional Imaging of Environmentally Critical Cyanobacteria Through Cryo-electron Tomography
}

\author{
Claire S. Ting*, Chyongere Hsieh**, Sesh Sundararaman*, Carmen Mannella** and Michael \\ Marko**
}

*Department of Biology, Williams College, 59 Lab Campus Drive, Williamstown, MA 01267 USA

** Resource for the Visualization of Biological Complexity, Wadsworth Center, Empire State Plaza, Albany, NY 12201 USA

Cyanobacteria of the genus Prochlorococcus are among the most abundant photosynthetic organis ms on Earth and contribute a significant fraction of the primary oxygen production in vast regions of the world's open oceans. Although Prochlorococcus shares close phylogenetic ties with marine Synechococcus and is found often in the same habitat, these cyanobacteria have evolved major differences in their genomes in response to selection pressures present in the ocean environment [1]. Through the use of scanning and conventional transmission electron microscopy, we have observed that these closely related cyanobacteria have also evolved significant differences in their cellular ultrastructure, particularly in the organization of the internal membranes on which photosynthesis occurs [2]. Detailed information about cellular organization needed to understand the molecular physiology of these cyanobacteria is currently lacking. The goal of this project is to conduct a comparative study of the 3-D architecture of these closely related cyanobacteria using electron tomography, focusing on the structure, organization, and contacts of the internal membranes. This is done in two steps. First, frozen-hydrated whole-cell mounts, shown here, are used to study the overall organization. Then, based on our experience with tissue [3,4], tomography of frozen-hydrated cryo-ultramicrotome sections is used to obtain higher-resolution information on membrane-associated macromolecules.

In order to visualize intracytoplasmic membranes and other cellular structures as close to their native state as possible, we preserved Prochlorococcus and Synechococcus cells by plunge freezing in liquid ethane at liquid nitrogen temperature. Tilt series ( $1^{\circ}$ increment, $120^{\circ}$ angular range) were recorded at $-178^{\circ} \mathrm{C}$ and $400 \mathrm{keV}$ acceleration voltage using a JEOL JEM4000FX with Gatan GIF2002 energy filter operated in zero-loss mode. The ice thickness was typically $0.6-1 \mu \mathrm{m}$.

In Figure 1, a conventional thin-section transmission electron micrograph of a chemically preserved Prochlorococcus cell (A) is shown along with a single zslice (1.8 nm thick) from a tomogram of a frozen-hydrated Prochlorococcus cell (B). The intracytoplasmic membranes are a dominant feature. In both chemically fixed and frozen-hydrated cells, these membranes are tightly appressed, and located near the cell periphery. A single phospholipid bilayer of the membrane is approximately 6 to $7 \mathrm{~nm}$ wide, and the width of an individual lamella consisting of two phospholipid bilayers and an intramembrane space ranges from $15 \mathrm{~nm}$ to $19 \mathrm{~nm}$. Additional cellular structures that are clearly visible in the frozen-hydrated Prochlorococcus cell include the cell wall, plasma membrane, and carboxysome.

Figure 2 shows zslices from tomograms of another Prochlorococcus isolate, MED4 (A), and from a marine Synechococcus isolate WH8102 (B). Comparisons of frozen-hydrated Prochlorococcus and Synechococcus cells confirmed our earlier observations from scanning electron micrographs on differences in cell size and cell morphology. Prochlorococcus MIT9313 (ca. 0.5 x $0.7 \mu \mathrm{m}$ ) is smaller than Synechococcus WH8102 (ca. 0.6 x $1 \mu \mathrm{m}$ ), but cells of both isolates are oval or rod-shaped. In contrast, Prochlorococcus MED4 cells are more spherical and are much smaller (ca. $0.4 \mu \mathrm{m}$, diameter) than MIT9313. 16S rDNA sequence analyses indicate that Prochlorococcus MIT9313 is more closely related to Synechococcus WH8102 than MED4, which is a more recently evolved Prochlorococcus strain. 
Striking differences are present in the cell walls of these cyanobacteria. In Synechococcus WH8102, the cell wall is thick and is associated with a prominent peptidoglycan layer between the outer and cytoplasmic membranes (Fig. 2B). In contrast, the cell walls of the two Prochlorococcus strains, particularly the more recently evolved MED4 isolate, are conspicuously thinner. Significant differences are also apparent in the structure and organization of the intracytoplasmic membranes. Compared to Prochlorococcus MIT9313 (Fig. 1A), the internal membranes of Synechococcus WH8102 (Fig. 2B) are not restricted to the cell periphery and are more widely spaced. Analysis of tomograms indicates that in Synechococcus, these internal membranes have several gaps or slots distributed around the cell. The cellular organization of the internal membranes is not identical between the two Prochlorococcus isolates. In MED4 (Fig. 2A), these membranes do not extend along the entire length of the cell periphery. The structural information provided by electron tomography has the potential to advance our fundamental understanding of the cellular organization of photosynthetic microorganisms, and in particular, of the three-dimensional structure of the photosynthetic lamellae. This in turn will contribute to our basic understanding of photosynthesis and membrane biogenesis in photosynthetic organisms. [5]

\section{References}

[1] C.S. Ting et al. Trends Microbiol. 10 (2002) 134.

[2] C.S. Ting et al. manuscript in preparation.

[3] C. Hsieh et al. J. Struct. Biol. 138 (2002) 63.

[4] C. Hsieh et al. Microsc. Microanal. 9 (Suppl. 2) (2004) 1178CD.

[5] Supported by Williams College Science Center Funds and Wadsworth's NIH / NCRR Biomedical Research Technology Program Grant RR01219 (PI J. Frank).
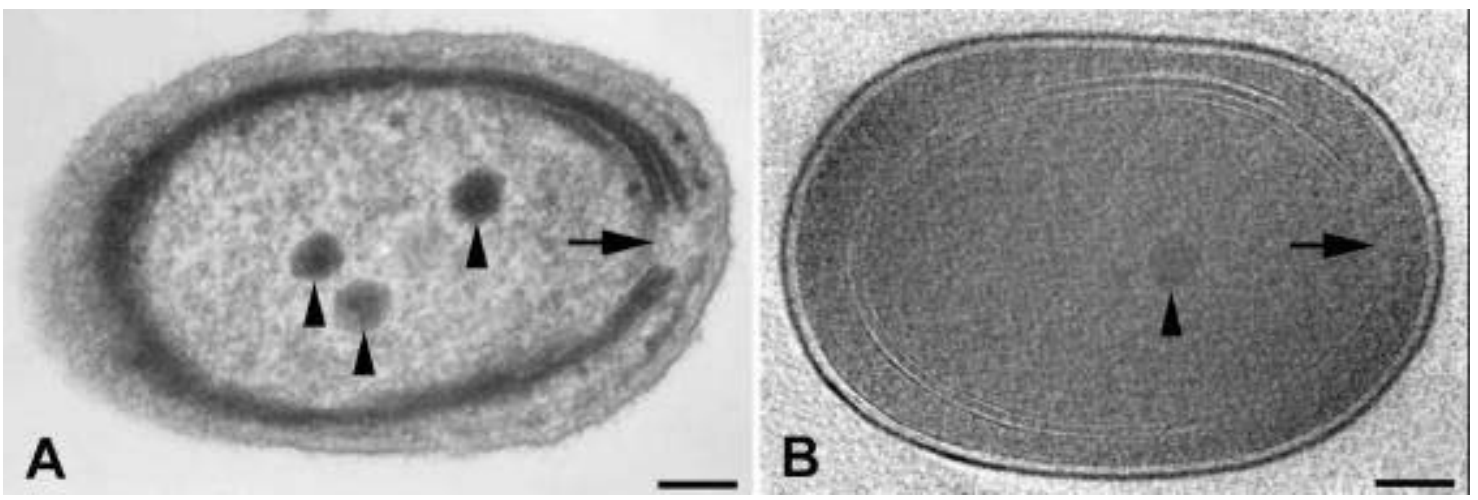

Figure 1. Comparison of Prochlorococcus MIT9313 cells preserved by chemical fixation (A) and by rapid freezing, as shown by a 1.8-nm z-slice through a tomogram of a frozen-hydrated cell (B). In Figs 1 and 2, note internal membrane gaps (arrows) and carboxysomes (arrowheads). Bars $=100 \mathrm{~nm}$.
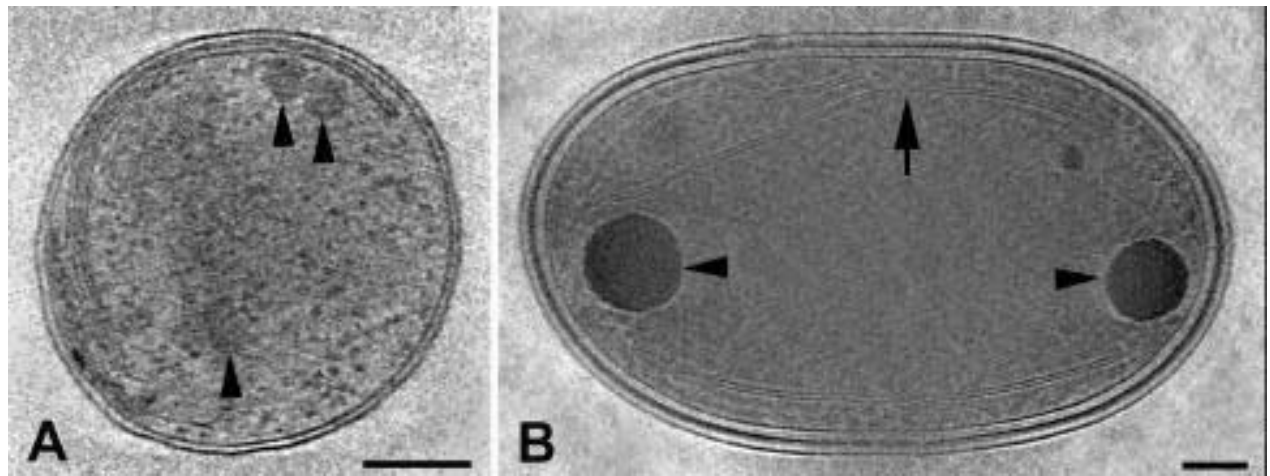

Figure 2. Frozen-hydrated Prochlorococcus MED4 (A) and Synechococcus WH8102 (B). Shown are $1.8 \mathrm{~nm}$ tomographic z-slices, with a cell thickness of $350 \mathrm{~nm}$ (A) or $600 \mathrm{~nm}$ (B). Bars $=100 \mathrm{~nm}$. 